\title{
Magnetic Moment of Heavy Baryons
}

\section{A.R.Haghpeima \\ Department of physics, Faculty of Sciences, Islamic Azad University, Mashhad Branch, Iran}

\section{Abstract:}

The baryon magnetic moment is a fundamental observable as its masswhich encodes information of the underlying quark-gluon structure and dynamics. Assuminga conventional correlated perturbative chiral quark model $(\mathrm{CP} \chi \mathrm{QM})$ we suggest that the charmedheavy baryons are a bound state of two light diquarks and a single heavy charm antiquark, the spatially wave function of these diquarks has a P- wave and an S-wave in angular momentum in the first and second version of our model respectively, as the result of these considerations we construct the orbital - flavor - spin symmetry of contribution of quarks. Then we calculate their magnetic moments in our model.

Key words: Heavy Baryon, Magnetic Moment, Quark Model,Quark Symmetry

\section{Introduction}

The magnetic moment is an intrinsic observable of particles which may encode important information of its quark-gluon structure and will help us deepen our understanding of the underlying dynamics. The heavybaryon masses and magnetic moments in several typical models have been calculated [1]. Now we calculate masses and magnetic moments for them using our diquark model. Previously we have used our vector diquark model for calculating the mass and stability / magnetic moment of Theta + pentaquark state.

Theoretically, the study of heavy baryons has always been interesting and these baryons play an important role in our understanding of QCD at the hadronic scale. There are many theoretical treatments of heavy baryons [2, 3, 4] including quark models, QCD sum rules, Lattice QCD [5, 6], the Relativistic quark-diquark approximation. Non-relativistic QCD, NRQCD which has been able to explain the mass spectrum of light baryons which is an effective field theory obtained from QCD by integrating out modes of an energy of the order of the heavy- quark masses for describing baryons made of one or more heavy quarks[7, 8].The heavy - quarklight diquark HQLD sector of NRQCD lagrangian is a heavy quark effective theory HQET. In this effective field theory framework, EFT of heavy baryons where the typical gluon momenta are small compared with the heavy quark mass $\mathrm{m}_{\mathrm{Q}}$, $\mathrm{QCD}$ dynamics of light diquark is independent of the flavor and spin of heavy quark. For the heavy flavors, this new symmetry called heavy quark symmetry, HQS. In fact, in this limit of heavy quark mass, low energy QCD dynamics remains non-perturbative but using HQS one can separate the light quark and gluon dynamics from that of heavy one by 
systematically expanding the QCD lagrangian in powers of $1 / \mathrm{mQ}$ and imposing HQS effects [9, 10, and 11]. According to these effects in heavy baryons, the light degrees of freedom quantum numbers are well-defined up to corrections in the inverse of the $\mathrm{m}_{\mathrm{Q}}$.Consequently, the heavy quark momentum is close to the kinetic momentum resulting from the hadrons motion. Thus the kinetic energy of the internal motion of the heavy baryon system is close to the kinetic energy of the relative motion of the heavy quark and light diquark up to corrections of the $\mathrm{m}_{\mathrm{L}} / \mathrm{m}_{\mathrm{Q}}$ where $\mathrm{L}$, denotes a light quark. This is one of the bases for treating the light quark subsystem as a diquark in our calculations. The quark-diquark picture of a heavybaryon is the nice approximation used to describe the baryon properties [12]. In this picture, we reduce the task of treating a three-body system to a two body system which is a successful task especially where we approximate the heavy quark massthe $\mathrm{m}_{\mathrm{Q}}$ to be infinity with respect to mass scale in the process, and hence enormously reduces the complexity of theoretical analysis. The paper is organized as follows. In the next section we introduce HQS effects for heavy baryons and calculate their mass spectrum using this symmetry limit.Finally, section 3 devoted to conclusions and results.

\section{HQS limit}

Theoretically, the full QCD Lagrangian for a heavy quark (c, b ort) is given by

$$
\mathrm{L}_{\mathrm{Q}}=\mathrm{Q}\left(\mathrm{i} \gamma_{\mu} \mathrm{D}^{\mu}-\mathrm{m}_{\mathrm{Q}}\right) \mathrm{Q}
$$

Where $D^{\mu} \equiv \partial^{\mu}-\operatorname{ig}_{s} T^{a} A^{a \mu}$ with $T^{a}=\lambda^{a} / 2$.Thus the heavy quark interacts with the light degrees of freedom by exchanging gluons with the momenta of order $\Lambda_{\mathrm{QCD}}$ which is much smaller than its mass $m_{Q}$. In the HQS limit with low energy situations, where the typical gluon momenta are small compared with the heavy quark mass $(\mathrm{MQ}), \mathrm{QCD}$ dynamics becomes independent of the heavy degrees of freedom, especially for the flavor and spin of the heavy quark. This means that the hyperfine interaction that involves the heavy quark is suppressed by the mass of the heavy quark. As a consequence, one-gluon exchange HF interaction should depend on the interacting light diquark pair, independently of the baryon the pair belongs to. In fact, the QCD hyperfine interaction andthe QED electromagnetic hyperfine interaction between $\mathrm{i}$ and $\mathrm{j}$ quarks are proportional to $1 / \mathrm{m}_{\mathrm{i}} \mathrm{m}_{\mathrm{j}}$, where $\mathrm{m}_{\mathrm{i}}, \mathrm{mj}$ are their masses. These interactions contribute to the systematic uncertainty of the experimental results and can be ignored in HQS limit, where one of the quarks is heavy [14]. Indeed we characterize the heavy baryon mass by two widely separated scales: the large heavy quark mass, $\left(\mathrm{m}_{\mathrm{Q}}\right)$, and the low momentum transfer between the heavy and the light quarks of the diquark, which is of order $\Lambda_{\mathrm{QCD}}$. In this system, the light diquark circle around the nearly static heavy quark and the system behave as the QCD analog of the familiar hydrogen bounded by electromagnetic force. In HQS limit, where $\mathrm{m}_{\mathrm{Q}} \rightarrow \infty$ a good quantum number is the angular momentum of the light degrees of freedom. Thus, heavy quark baryons belong to either SU (3) antisymmetric $3_{\mathrm{F}}$ or symmetric $6_{\mathrm{F}}$ representations fig.1. The spin of the light diquark is 0 for $3_{\mathrm{F}}$, while it is 1 for $6_{\mathrm{F}}$ For the spin of the ground state heavy baryons we have $1 / 2$ for $3 \mathrm{~F}$, representing the $\Lambda \mathrm{h}$ and $\Xi$ h heavy baryons, while it can be both $1 / 2$ or $3 / 2$ for 
$\sigma_{\mathrm{F}}$, representing $\Sigma^{\mathrm{h}}, \Sigma^{\mathrm{h}^{*}}, \Xi^{\mathrm{h}}, \Xi^{\mathrm{h}^{*}}, \Omega^{\mathrm{h}}$ and $\Omega^{\mathrm{h}^{*}}$, where the star and $\mathrm{h}$ indicates spin $3 / 2 \mathrm{c} \mathrm{b}$ quarks respectively. The mass difference between states belonging to different representations $3_{\mathrm{F}}$ and $6_{\mathrm{F}}$, do contain the dynamics of the light scalar and vector diquark subsystem respectively. But the mass splitting between states belonging to same representation is caused by the chromomagnetic interaction at the order $1 / \mathrm{mQ}$ and can be ignored in HQS limit. Thus baryons containing a single heavy quark should fall into almost degenerate multiplets.For example, there is no mass difference between $\mathrm{Q}^{*}$ and $\mathrm{Q}$ heavy baryons for $6_{\mathrm{F}}$. Generally, these states have the same parity as the light component.

\section{Fig 1:SU (3) multiplets of charmed baryons, (a) 3F antisymmetric and (b) 6F symmetric}

\section{Representations.}

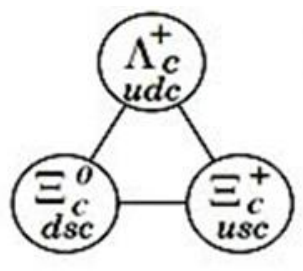

(a)

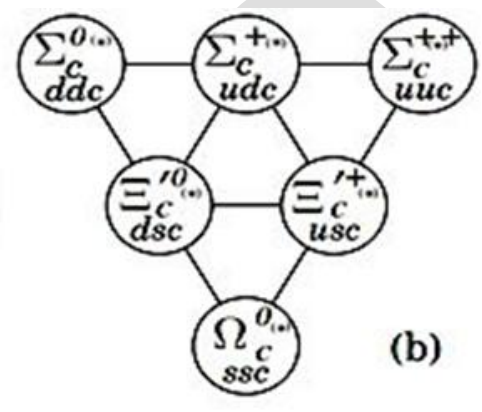

(b)

The members of the two multiplets of singly charmed baryons have flavor wave functions

$$
\begin{aligned}
& \Sigma^{\mathrm{c}++}=\text { uuc, } \Sigma \mathrm{c}^{+}=1 / \sqrt{2}(\mathrm{ud}+\mathrm{du}) \mathrm{c}, \Sigma \mathrm{c} 0=\mathrm{ddc} \\
& \Xi^{\mathrm{c}+}=1 / \sqrt{2}(\mathrm{us}+\mathrm{su}) \mathrm{c} \Xi^{\prime c 0}=1 / \sqrt{2}(\mathrm{ds}+\mathrm{sd}) \mathrm{c} \\
& \Omega^{\mathrm{c}}=\mathrm{ssc},
\end{aligned}
$$

For the sextet and

$$
\begin{gathered}
\Lambda^{\mathrm{c}+}=1 / \sqrt{2}(\mathrm{ud}-\mathrm{du}) \mathrm{c}, \Xi^{\mathrm{c}+}=1 / \sqrt{2}(\mathrm{us}-\mathrm{su}) \mathrm{c} \\
\Xi^{\mathrm{c} 0}=1 / \sqrt{2}(\mathrm{ds}-\mathrm{sd}) \mathrm{c}
\end{gathered}
$$

For the antitriplet whichare similarto theset of flavorwave functions for baryons containing b quark.

Table 1:The s-wave heavy baryons and their quantum numbers.

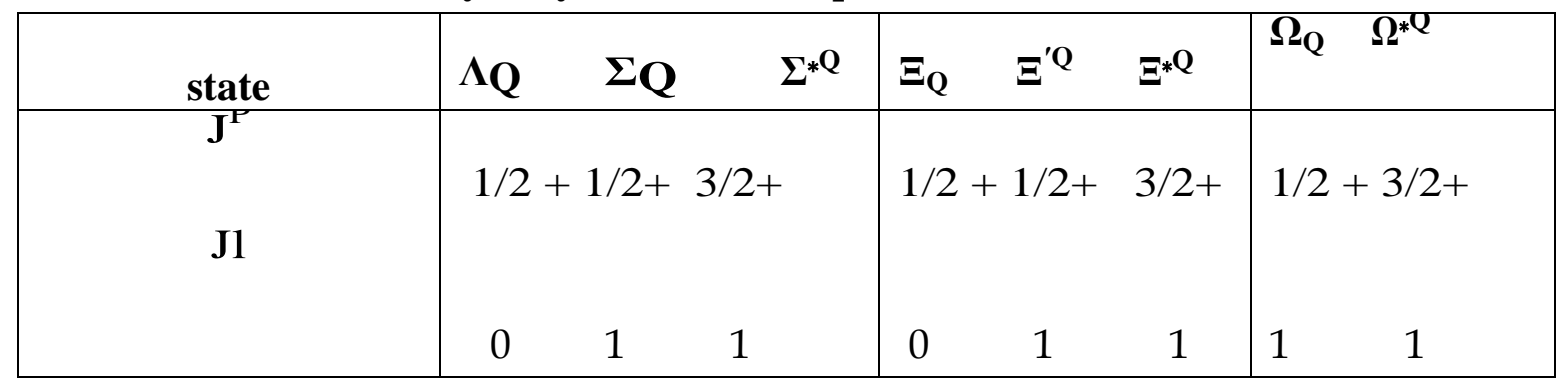

Table 2, shows the experimental masses of the Ground-state charmed and bottom 
baryons [15].

Table 2: Ground - state charmed baryons and their SU (3) multiplets

Lattice estimates $(\dagger)$ have been taken from (Ref [16]).

\begin{tabular}{|c|c|c|}
\hline Heavy baryon & $\operatorname{Mass}(\mathrm{GeV})$ & SU(3)multiplet \\
\hline 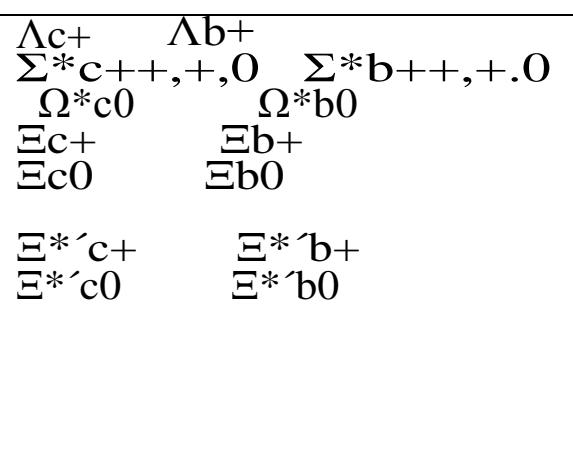 & $\begin{array}{c}2.285-5.624 \\
2.455-5.808 \\
\\
2.698-5.990^{\dagger} \\
2.468-5.793 \\
2.471-5.760^{\dagger} \\
\\
2.576-5.900^{\dagger} \\
2.578-5.900^{\dagger}\end{array}$ & $\begin{array}{l}3 \text { bar } \\
6 \\
6 \\
3 \text { bar } \\
3 \text { bar }\end{array}$ \\
\hline
\end{tabular}

In the limit of HQS, where the heavy quark mass $\mathrm{m}_{\mathrm{Q}} \rightarrow \infty$, all states in the $6 \mathrm{~F}$ representation would be degenerate and this is true for all states in the $3_{\mathrm{F}}$ representation. In this limit, without the $\mathrm{m}_{\mathrm{Q}} \rightarrow \infty$ approximation there is a mass splitting between states belonging to each representation due to differences between the masses of the light diquark sectors of the heavy baryons. We calculated the light diquark masses by adding the two quarks mass and their binding hyperfine HF energy. Table .3.

Table 3: Quark and diquark masses and quantum numbers.

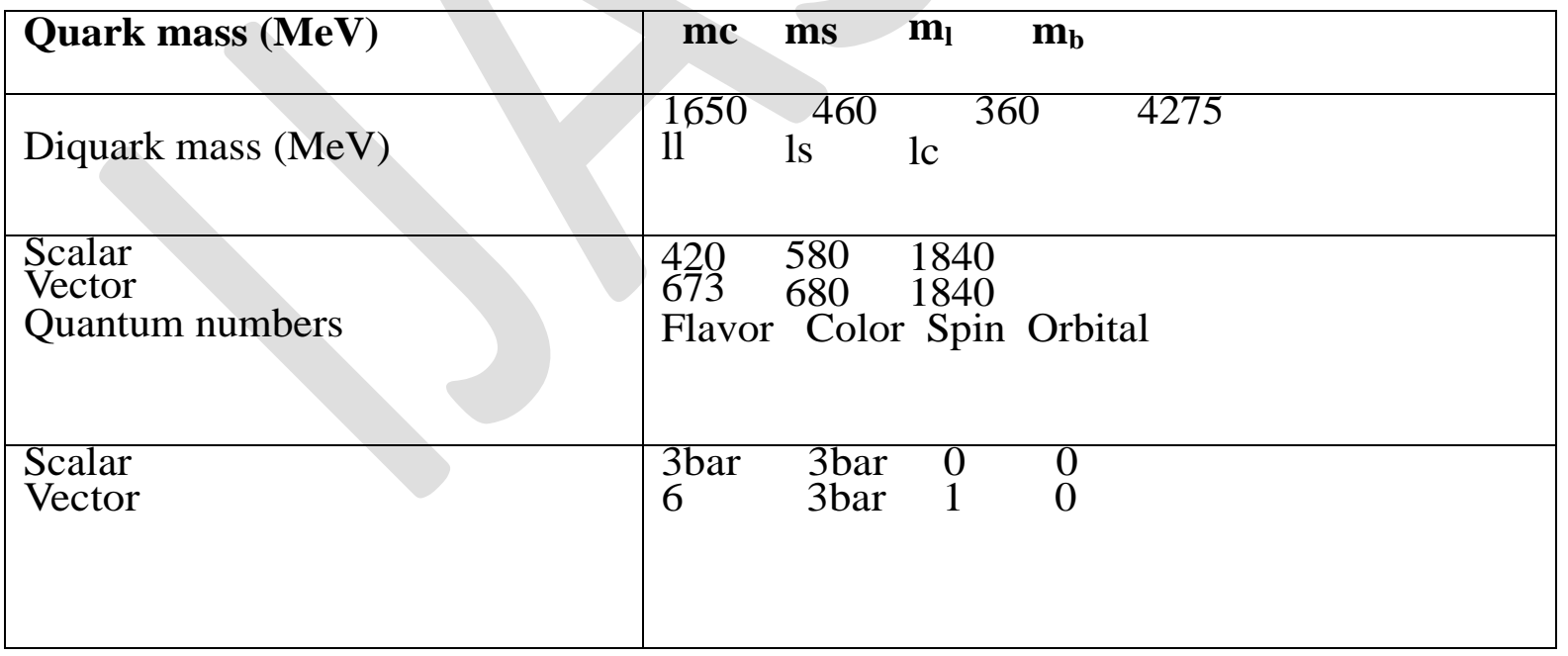

Now we evaluate the masses of the ground state heavy baryons in the framework of the HQS limit. Thus we can use the mass formula

$$
M=m_{D}+m_{Q}+E_{L}+E r
$$

Here, $\mathrm{m}_{\mathrm{D}}$ is the light diquark mass, $\mathrm{m}_{\mathrm{Q}}$ the heavy baryon mass, $\mathrm{E}_{\mathrm{L}}$ the orbital and $\mathrm{Er}$ 
the radial exciting energies between heavy quark and light diquark respectively. According to table 3 two quarks having a closer mass have more tightly bound which is indicated by the spin-spin interaction, thus the mass splitting

$$
(\mathrm{ud})-[\mathrm{ud}]>(\mathrm{us})-[\mathrm{us}]>(\mathrm{uc})-[\mathrm{uc}] \simeq 0
$$

Is expected where [ ], (), denotes scalar and vector diquarks respectively. We have accommodated the ground state, $\mathrm{J}^{\mathrm{P}}=1 / 2^{+}$heavy charmed and bottom baryons. These states have no orbital angular momentum, $\mathrm{E}_{\mathrm{L}}=0$ and the mass splitting between them is indicated by radial exciting energy, Er of each ground state heavy baryon. By using this exciting energy we have evaluated the average distance between heavy quark and the center of mass of the light diquark for each heavy baryon state. We set the Jacobi coordinates for a heavy quark -light diquark description.fig. 2.

\section{Fig 2: Q2q rest frame}

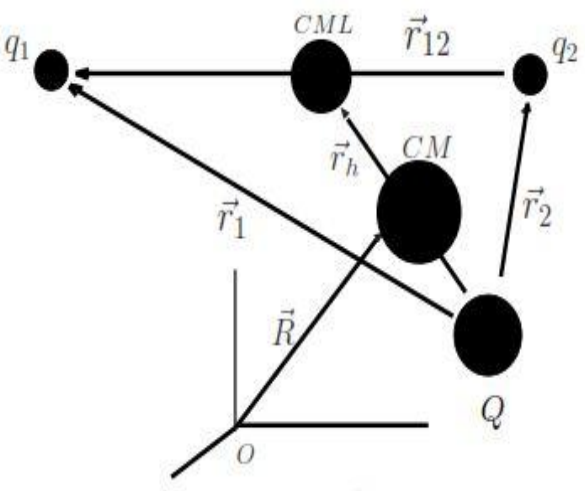

For the coordinates we consider the following relations

$\mathrm{R}=\mathrm{m}_{\mathrm{q} 1} \mathrm{x}_{\mathrm{q} 1}+\mathrm{m}_{\mathrm{q} 2} \mathrm{x}_{\mathrm{q} 2}+\mathrm{m}_{\mathrm{Q}} \mathrm{x}_{\mathrm{h}} / \mathrm{m}_{\mathrm{q} 1}+\mathrm{m}_{\mathrm{q} 2}+\mathrm{m}$

$\mathrm{r}_{12}=\mathrm{x}_{\mathrm{q}}(6)$

$\mathrm{r}_{\mathrm{h}}=\left(\mathrm{m}_{\mathrm{q} 1} \mathrm{x}_{\mathrm{q} 1}+\mathrm{m}_{\mathrm{q} 2} \mathrm{x}_{\mathrm{q} 2} / \mathrm{m}_{\mathrm{q} 1}+\mathrm{m}_{\mathrm{q} 2}\right)-\mathrm{x}_{\mathrm{h}}$

Where $\mathrm{x}_{\mathrm{q} 1}, \mathrm{x}_{\mathrm{q} 2}$, and $\mathrm{x}_{\mathrm{h}}$ represent the positions with respect to a certain reference frame and $r_{12}$ and $r_{h}$ are the Jacobian coordinates. Thus we would have the heavy baryon Kinetic energy

$$
\mathrm{T}\left(\mathrm{q}^{2} \mathrm{Q}\right) \simeq \nabla_{\mathrm{rh}}^{2} / 2 \mu
$$

Where $\nabla^{2}$ denotes the Laplacian and $\mu$ are the heavy quark-light diquark reduced mass. By using the Baryon wave function

$$
\Psi_{B}=N\left[Y_{00}(r h) \exp \left(-a^{2} r_{h}^{2} / 2\right)\right](8)
$$

We would have the Kinetic energy

$$
\mathrm{Er}=<\mathrm{T}>\psi \simeq 3 \mathrm{a}^{2} / 4 \mu
$$


And for the relative distance between heavy quark and light diquark we have

$$
\mathrm{r}_{0}=\left\langle\mathrm{r}_{\mathrm{h}}>=\sqrt{5} / 2 \mathrm{a}^{2}(10)\right.
$$

We have calculated the radial kinetic energy, Er of each ground state heavy baryon listed in table 2, using their parameters, $m_{D} m_{Q}$ and $E r=0$. Also by using of Eq8-9, we obtained the average distance, $r_{0}$ between the heavy quark and the center of mass of light diquark. Table 4.

The results with QCD sum rule[ 16 ] and lattice QCD calculation [ 17 ] have suggested a clear dominance of the collinear-type configurations ( the heavy quark is close to the center of mass of the light diquark).This result seems to support our calculations based on HQS limit of HQLD picture of heavy baryons. In Ref. [18], the authors studied the baryon properties using Isgur-Wise function and found the heavy quark is far from the light diquark which is against the HQS approximation of HQLD.

Table 4: Ground - state charmed and bottom baryons and their radial kinetic energy and the relative distance between heavy quark and light diquark center of mass, Experimental masses have been taken from ( Ref [17] ) and Lattice estimates ( $\dagger$ ) have been taken from ( Ref [16] ).

\begin{tabular}{|c|c|c|c|}
\hline Heavy baryon & Mass $(\mathbf{G e V})$ & $\mathbf{E}_{\mathbf{r}}(\mathbf{M e V})$ & $\mathbf{r}_{\mathbf{0}}(\mathbf{M e V})^{-\mathbf{1}}$ \\
\hline$\Lambda \mathrm{c}+\Lambda \mathrm{b}+$ & $2.285-5.624$ & $215-929$ & $0.00509-0.00229$ \\
$\Sigma \mathrm{c}++*,+, 0 \Sigma^{*} \mathrm{~b}++,+, 0$ & $2.455-5.808$ & $132-860$ & $0.00535-0.00192$ \\
$\Omega^{*} \mathrm{c} 0 \Omega^{*} \mathrm{~b} 0$ & $2.698-5.990$ & $368-103$ & $0.00320-0.00174$ \\
$\Xi \mathrm{c}+\Xi^{+} \Xi^{*} \mathrm{c} 0$ & $2.468-5.793$ & $238-938$ & $0.00425-0.00179$ \\
$\Xi \mathrm{b} 0 \Xi^{*} \mathrm{c}+\Xi^{*} \mathrm{~b}+$ & $2.471-5.760$ & $241-905$ & $0.00423-0.00200$ \\
$\Xi^{*} \mathrm{c} 0 \Xi^{*} \mathrm{~b} 0$ & $2.576-5.900$ & $246-945$ & $0.00392-0.00183$ \\
& $2.578-5.900$ & $248-945$ & $0.00391-0.00183$ \\
& & & \\
\hline
\end{tabular}

The average size ofa scalar anda vector diquark is $0.0045 \mathrm{MeV}^{-1}$ and $0.0205(0.0235)$ $\mathrm{MeV}^{-1}$ respectively. According to Table 4 one sees that the average distance of the heavy quark to the center of mass of the light diquark, $r^{0}$ is smaller than the average size of the light diquark.The picture that emerges from this analysis is the one depicted in Fig.3, where the heavy quark is too close to the center of mass of the light diquark, which is in agreement with the findings of Ref [18].

Fig 3:schematic picture of a Ground-state spin 1/2 heavy baryon with a charmed heavy quark (a), and a bottom heavy quark (b).
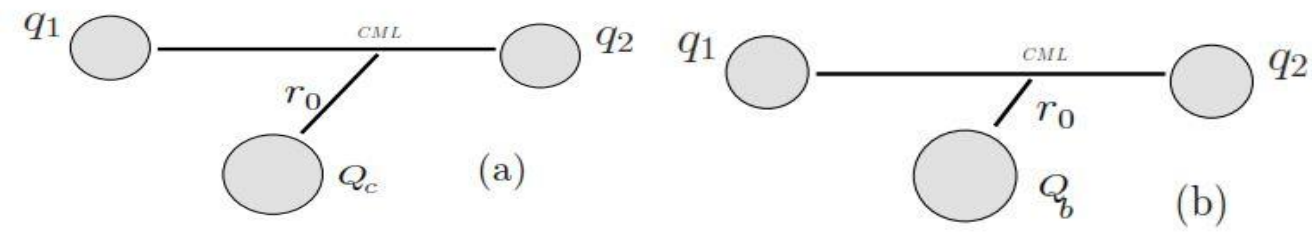
These findings based on HQS limit of HQLD approximation shows a dominance of collineartype configuration, which confirms the results of QCD sum rules[16] and lattice calculations [17].We have obtained the average distance, $\mathrm{r}^{0^{*}}$ between the heavy quark and the center of mass of light diquark for charmed and bottomed baryons with spin 3/2 Table .5. One sees that this average distance for the spin 3/2 state heavy baryons is smaller than the spin $1 / 2$ states. This distance splitting between states belonging to same representation is caused by the chromomagnetic interaction and usually can be ignored in HQS limited with $\mathrm{M}_{\mathrm{Q}} \rightarrow \infty$ approximation. The picture is depicted in Fig.4.

Fig 4:schematic picture of a Ground-state spin 3/2heavy baryon with a charmed heavy quark (a) and a bottom heavy quark (b).

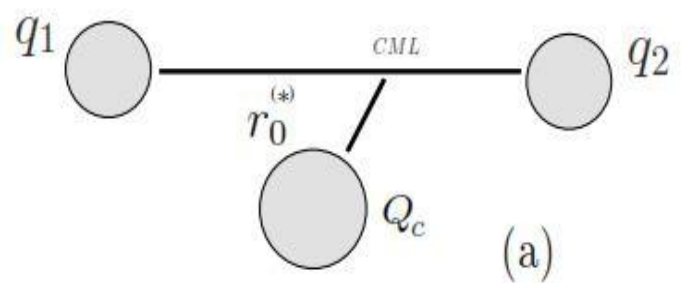

(a)

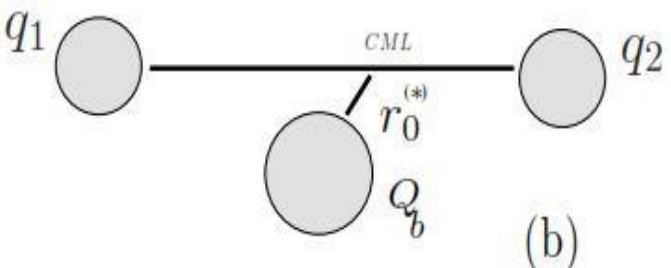

Table

$3 / 2$, theirmasses (Ref [16-17] their SU (3) multiplets and the relative distance $\mathbf{r}_{0} *$, between heavy quark and light diquark center of mass.

\begin{tabular}{|l|l|l|l|}
\hline Heavy baryon & Mass $(\mathbf{G e V})$ & $\begin{array}{c}\text { SU(3) } \\
\text { multiplet }\end{array}$ & $\mathbf{r}_{\mathbf{0}}\left(\mathrm{MeV}^{-\mathbf{1}}\right)$ \\
\hline$\Sigma_{\mathrm{c}}^{++,+, 0^{*}} \Sigma_{\mathrm{b}}^{++,+, 0^{*}}$ & $2.518-5.833$ & 6 & $0.00477-0.00190$ \\
$\Omega_{\mathrm{c}^{*}}{ }^{0} \Omega_{\mathrm{b}^{*}}^{0}$ & $2.768-6.000$ & 6 & $0.00297-0.00162$ \\
$\Xi_{\mathrm{c}}^{+0^{*}} \quad \Xi_{\mathrm{b}}^{\prime}{ }^{+0^{*}}$ & $2.646-5.900$ & 6 & $0.00350-0.00180$ \\
\hline
\end{tabular}

\section{Magnetic Moment Calculation}

For the magnetic moment of a particle we have:

$$
\vec{\mu}=g \vec{S}
$$

Where $\mu$ is the magnetic moment, $g$ is gyromagnetic ratio and Sis the spin operator, this leads to, $\mu_{z}=g S_{z}$. For the quarks we have:

$$
g_{q}=g_{s} \mu_{q}=2 \mu_{q}=2 \frac{Q_{q}}{2 m_{q}}=\frac{Q_{q}}{m_{q}}
$$

In which $\mu_{q}$ is quark magneton, and $Q_{q}, m_{q}$ are quarks charge and mass respectively.

If the particle has angular momentum, themagnetic moment would be: 


$$
\vec{\mu}=g \vec{S}+g_{l} \vec{l}
$$

We conclude that for the heavy baryon we have:

$$
\mu_{z}=\left\langle\psi_{f s}\left|\sum_{i} g_{i} S_{z}^{i}+g_{l_{i}} l_{z}^{i}\right| \psi_{f s}\right\rangle
$$

In which $\Psi_{f s}$ is the flavor- spin wave function of the heavy baryon. The contribution of the second term would be zero. Table 6 shows our magnetic moment calculations using flavor wave functions $\mathrm{Eq}(2,3)$ and similar triplet and singlet spin wave functions for the scalar and vector light diquarks in our model. The spin of the light diquark is 0 for $3_{\mathrm{F}}$, while it is 1 for $6_{\mathrm{F}}$. For the spin of the ground state heavy baryons we have $1 / 2$ for $3 \mathrm{~F}$ and $1 / 2,3 / 2$ for $6_{\mathrm{F}}$.

Table 6: Magnetic moments of heavy baryons $(Q=b, c)$

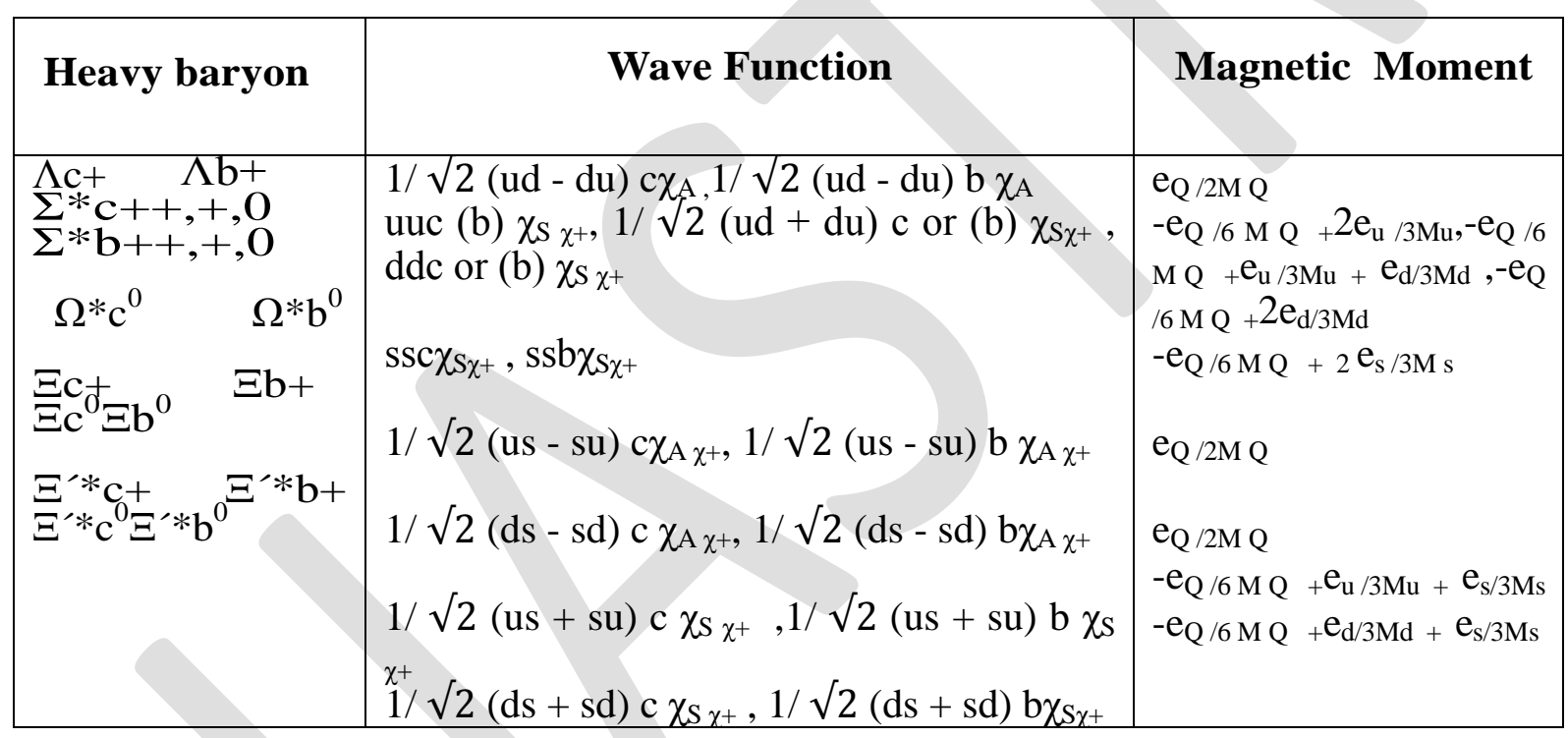

\section{CONCLUSION}

We calculated the masses and magnetic moments of charmed and bottomed heavy baryons for $3_{\mathrm{F}}$ and $6_{\mathrm{F}}$ multiplets with single heavy $\mathrm{b}$ or $\mathrm{c}$ quark in the framework of heavy quark symmetry limit using our distance configuration approach in perturbative chiral quark model and compared the results with the existing predictions in the literature. Our results on masses and magnetic moments of heavy baryons are in good agreement with many results listed in the literature [19] and may be checked via different non-perturbativeapproaches. Alsochecking our results by future experiments can be useful for understanding the internal structure as well as the geometric shape of these baryons. 


\section{REFERENCES}

[1] A. Ocherashvili et al., SELEX Collaboration, Phys. Lett. B 628, 18 (2005). J. Eigelfried et al., SELEX Collaboration, Nucl. Phys. A 752, 121 (2005). Zalak Shah, Kaushal Thakkar, Ajay Kumar Rai and P. C. Vinodkumar, arXiv: 1602.06384v2 [hep-ph] (2016). Kaushal Thakkar and P. C. Vinodkumar, proceedings of Science Hadron 2013, 055 (2013). Ajay Majethiya, Bhavin Patel, P. C. Vinodkumar Eur. Phys. J. A 38:307-315 (2008). D. Ebert, R. N. Faustov and V. O. Galkin, Phys. Rev. D 84, 014025 (2011). H.-X. Chen, W. Chen, Q. Mao, A. Hosaka, et al., Phys. Rev. D 91, 054034 (2015). T. Yoshida, E. Hiyama, A. Hosaka, M. Oka, and K. Sadato, Phys. Rev. D 92, 114029 (2015).

[2] Chien-WenHwang, J.Phys.G: Nucl. Part.Phys. 35, 075003(2008). Garcilaso et al., J. Phys.G: Nucl. Part. Phys. 34, 961-976(2007). S.Migura, D.Merten, B.Metsch and H.R.Petry, Eur.Phys. J. A 28, 41 (2006).

[3] S.M.Gerasyuta and D.V.Ivanov, Nuovo Cim.A112, 261 (1999). D.Ebert, R.N. Faustov, V.O.Galkin and A.P. Martynenko, Phys. Rev. D 66, 014008 (2002). S. Migura, D. Merten, B. Metsch and H.R. Petry, Eur. Phys. J. A 28, 41 (2006). C. Albertus, E. Hernandez, J. Nieves and J. M. Verde-Vel ASCO, Eur. Phys. J. A 32, 183 (2007). 8 Ashvin G.Godghate, Rajaram S.Sawant and Shobha D.Jadhav, An Evaluation of Physico-chemical Parameters to Assess Borewell Water Quality from Medical and Vadgaon Villages of Kagal Tahsil, MS, India, Int. Res. J.Environment Sci., 2(5), 95-97 (2013).

[4] E. Bagan, M. Chabab, H. G. Dosch and S. Narison, Phys.Lett. B 287, 176 (1992). D.W.Wang and M. Q. Huang, Phys. Rev. D67, 074025 (2003). X. Liu, H. X. Chen, Y. R. Liu, A. Hosaka and S. L. Zhu, Phys.Rev. D 77, 014031 (2008).

[5] A. Ali Khan et al., Phys. Rev. D 62, 054505 (2000). R. Lewis, N. Mathur, and R. M. Woloshyn, Phys. Rev D64, 094509 (2001). J. M. Flynn, F. Mescia, and A. S. B. Tariq [UK QCD Collaboration], JHEP 0307, 066 (2003).

[6] S. Capstick and N. Isgur, Phys. Rev. D 34, 2809 (1986). 4 V.E. Lyubovitskij et al., Prog. Part. Nucl. Phys. 50, 329 (2003); M.A. Ivanov, M.P. Locher, and V.E. Lyubovit- skij, Phys.Lett.B 408, 435 (1997); M.A. Ivanov and P.Santorelli, Phys. Lett. B456, 248 (1999). D. Ebert, R. N. Faustov, and V. O. Galkin, Phys. Rev. D 72, 034026 (2005). D. Ebert, R. N. Faustov, and V. O. Galkin, Phys. Lett. B 659, 612 (2008).

[7] A.Valcarce et al., Phy. Rev. C.72, 025206 (2005).

[8] W. E. Caswell and. P. Lepage, Phys. Lett. B167, 437 (1986); G. T. Bodwin, E. Braaten and G. P. Lepage, Phys. Rev. D51 , 1125 (1995) [Erratum-ibid. D 55 , 5853 (1997)] [arXiv:hep-ph/9407339].

[9] N. Isgur and M. B. Wise, Phys. Lett. B 232, 113 (1989); B237, 527 (1990) H. Georgi, Nucl. Phys. B 240, 447 (1990).

[10] S. Nussinov, and W.Wetzel, Phys. Rev. D 36, 130 (1987). M. A. Shifman, and M. B.Voloshing, Yad. Fiz. 45,463 (1987) [Sov. J. Nucl. Phys. 45, 292 (1987)]. H. D. Politzer, and M. B.Wise, Phys. Lett. B 206, 681 (1988); ibid. 208, 504 (1988). N. Isgur, and M. B. 
Wise, Phys. Lett. B 232, 113 (1989). M. Neubert, Phys. Rep. 245, 259 (1994). J.G.K“orner, M.Kr"amer, and. Pirjol, Prog.Part.Nucl. Phys. 33, 787 (1994).

[11] H. Georgi, Nucl. Phys. B 240, 447 (1990). M. Neubert, Phys. Rep. 245, 261 (1994). T. Mannel, W. Roberts, and Z. Ryzak, Nucl. Phys. B 368, 204 (1992). C. V. Efimov, M. A. Ivanov, N. B. Kumanovo, and V. E. Lyubo Novitski, Z. Phys.C 54, 349 (1992). X. - H. Guo, and P. Kroll, Z. Phys. C 59, 567 (1993). X. - H. Guo, and T. Muta, Phys. Rev. D 54, 4629 (1996), Mod. Phys. Lett. A 11, 1523 (1996). B. K“onig, J. G. K“orner, M. Kr“amer, and P. Kroll, Phys. Rev. D 56, 4282 (1999).

[12] M.Anselminoetal., Rev. Mod. Phys. 65, 1199 (1993). D. Ebert, H.Reinhard and M. K. Volkov, Prog. Part. Nucl.Phys. 33, 1 (1994). J. G. K“orner, M. Kr"amer and D. Pirjol, Prog. Part. Nucl. Phys. 33, 787 (1994). R. L. Jaffe, Phys. Rep.409, 1 (2005). F. Wilczek, hep-ph / 0409168.

[13] J. G. Korner, M. Kramer and D. Pirjol, Prog. Part. Nucl. Phys.33, 787 (1994).

[14] T.Altonen (CDFCollaboration), Phys. Rev. Lett. 99, 202001 (2007). C. Amsler (Particle Data Group), Phys. Lett.B 667, 1 (2008). J. L. Rosner, Phys. Rev. D 75, 013009 (2007).

[15]CDF collaboration, T.Aaltonen, Phys. Rev. Lett.99, 052002 (2007). D0Collaboration, V.Abazov, Phys. Rev. Lett.99, 052001 (2007).

[16] UK QCD Collaboration, K. C. Bowler, Phys. Rev. D 54, 3619 (1996).

[17] Particle Data Group, W. - M. Yao, J. Phys. G: Nucl. Part. Phys. 33, 1 (2006). BABAR Collaboration, B. Aubert ET. Phys. Rev. Lett. 97, 232001 (2006). CDF Collaboration, T.Aaltonen, Phys. Rev

[18] F. Cardarelli, S. Simulate, Phys. Lett. B 421, 295 (1998).

[19] Zalak Shah, Kaushal Thakkar, Ajay Kumar Rai and P. C. Vinodkumar, arXiv: 1602.06384v2 [hep-ph] (2016). 6. Kaushal Thakkar and P. C. Vinodkumar, proceedings of Science Hadron 2013, 055 (2013). 7. Ajay Majethiya, Bhavin Patel, P.C.Vinodkumar Eur. Phys. J. A 38:307-315 (2008). 8. D. Ebert, R. N. Faustov and V. O. Galkin, Phys. Rev. D 84, 014025 (2011). 9. H.-X. Chen,W. Chen, Q. Mao, A. Hosaka, et al., Phys. Rev. D 91, 054034 (2015). 10. T. Yoshida, E. Hiyama, A. Hosaka, M. Oka, and K. Sadato, Phys. Rev. D 92, 114029 (2015). 